\title{
SIMULTANEOUS ANALYTICAL METHOD DEVELOPMENT OF 6-MERCAPTOPURINE AND 6-METHYLMERCAPTOPURINE IN DRIED BLOOD SPOT USING ULTRA PERFORMANCE LIQUID CHROMATOGRAPHY TANDEM MASS SPECTROMETRY
}

\author{
MARLINA IKA ${ }^{1}$, RIZKA ANDALUSIA ${ }^{2}$, SUPANDI SUPANDI ${ }^{1}$, YAHDIANA HARAHAP ${ }^{1 *}$ \\ ${ }^{1}$ Department of Pharmacy, Faculty of Pharmacy, Universitas Indonesia, Depok, Indonesia. ${ }^{2}$ Department of Research and Development, \\ Dharmais Cancer Hospital, Jakarta, Indonesia. Email: yahdiana03@yahoo.com
}

Received: 21 April 2017, Revised and Accepted: 18 August 2017

\section{ABSTRACT}

Objective: 6-mercaptopurine (6-MP) is a chemotherapeutic agent in the antimetabolite class. It has to go through the metabolic pathway to form 6-methyl MP (6-MMP). This study aimed to obtain an optimum and validated method for the analysis of 6-MP and 6-MMP in dried blood spot (DBS) samples simultaneously and to evaluate the potential for future drug concentration monitoring in DBS samples.

Methods: The quality control and calibration curves were made by spotting $40 \mu \mathrm{L}$ blood on DBS paper and dried for 3 hrs. DBS papers were cut with a diameter of $8 \mathrm{~mm}$ and extracted with acetonitrile-methanol (1:3) containing internal standard 5-fluorouracil (5-FU). Separation was performed with waters acquity ultra performance liquid chromatography BEH C18 column of $1.7 \mu \mathrm{m}(2.1 \times 100 \mathrm{~mm})$ with a mobile phase consisting of $0.1 \%$ formic acid in water $0.1 \%$ formic acid in acetonitrile with gradient elution and a flow rate of $0.2 \mathrm{~mL} /$ minute. Mass detection was performed using Waters Xevo TQD with positive electrospray ionization (ESI) for 6-MP and 6-MMP and negative ESI for 5-FU in the multiple reaction monitoring mode.

Results: The detection rates of 6-MP, 6-MMP, and 5-FU were 153.09>119.09, 167.17>126.03, and 129.09>42.05, respectively. This method was linear with the range at $26-1000 \mathrm{ng} / \mathrm{mL}$ for 6-MP and $13-500 \mathrm{ng} / \mathrm{mL}$ for 6-MMP with consecutive $\mathrm{r} \geq 0.998$ and $\geq 0.999$, respectively. The $\%$ relative error value and $\%$ relative standard deviation for accuracy and precision of intraday and interday were not more than $15 \%$ and not more than $20 \%$ at the lower limit of quantification concentration, respectively.

Conclusions: This method fulfilled the requirements of selectivity, linearity, carry over, and matrix effects referring to the European Medicines Agency guidelines.

Keywords: 6-Mercaptopurine, 6-Methylmercaptopurine, Dried blood spot, Ultra performance liquid chromatography tandem mass spectrometry, Validation.

(C) 2017 The Authors. Published by Innovare Academic Sciences Pvt Ltd. This is an open access article under the CC BY license (http://creativecommons. org/licenses/by/4. 0/) DOI: http://dx.doi.org/10.22159/ijap.2017.v9s1.80_87

\section{INTRODUCTION}

6-mercaptopurine (6-MP) is an antimetabolite chemotherapy agent commonly used for acute lymphocytic leukemia (ALL). It is a prodrug that must be converted into its nucleotide by intracellular enzyme to cause a cytotoxic effect [1]. 6-MP has three major metabolic pathways. The first pathway is through the enzyme hypoxanthine-guaninephosphoribosyltransferase to form its active metabolite, 6-thioguanine nucleotide; the second pathway is through the enzyme S-thiopurine methyltransferase (TPMT) to form 6-methyl MP (6-MMP); and the third pathway is through the enzyme xanthine-dehydrogenase to form 6-thiouric acid [1].

6-MMP plays a role in causing the hepatotoxic side effects in patients given ALL chemotherapy [2]. Therefore, therapeutic drug monitoring of 6-MMP is required to ascertain that its concentration is in a safe range. The standard 6-MP dosage given to children suffering from ALL is $50-75 \mathrm{mg} / \mathrm{m}^{2}$, a variation that would appear to depend on body surface area [3,4]. 6-MP also displays a range of possible adverse drug reactions and a narrow therapeutic index; hence, the therapeutic index for each individual needs to be monitored [5].

Dried blood spot (DBS) is a bio sampling method recently developed for therapeutic drug monitoring. Patient blood is taken by a prick at the tip of a finger to produce a drop of blood which is spotted and dried on a special paper before analysis. This method has the advantage of minimum pain for patients, as the blood is taken using a sterile needle lancet on a finger, toe, or heel. Another advantage is the small amount of blood is taken $(10-80 \mu \mathrm{L})$. DBS is also convenient in respect of storage and distribution, while analysis of a sample of dried blood is relatively stable and this procedure reduces the risk of infection for the subject [6-8]. An analytical method for 6-MP and 6-MMP in plasma as well as in whole blood has already been developed; it uses high-performance liquid chromatography (HPLC) with the ultraviolet detector and ultra-PLC tandem mass spectrometry (UPLC-MS/MS) [5,9]. The experimental analysis of 6-MP and 6-MMP in a DBS sample using UPLC-MS/MS presented here is believed to be the first such conducted.

This research aimed to obtain a valid analytical method of testing 6-MP and 6-MMP simultaneously in a DBS sample with UPLC-MS/MS using 5 -fluorouracil (5-FU) as the internal standard. The sample preparation and extraction were performed using a mixture of acetonitrile and methanol. Method optimization and validation were aimed at to meet the requirements refer to the European Medicines Agency (EMA). This method is expected to be applicable to the monitoring of the drug therapy of 6-MP in infant patients with ALL.

\section{METHODS}

Chemicals

Acetonitrile HPLC grade, formic acid, and methanol HPLC grade were obtained from Merck. 6-MP and 6-MMP were from Sigma. All water was HPLC grade and prepared using a Millipore Direct-QTM 5 water system (Millipore, Watford, UK) and filtrated using Sartorius membrane filters (0.45_m) obtained from Sartorius (Epsom, UK). Whole blood was from the Indonesian Red Cross. 
Stock solutions, standards, and quality controls

Standard stock solutions of $1 \mathrm{mg} / \mathrm{mL}$ were freshly prepared by separately dissolving 6-MP, 6-MMP, and 5-FU into $2 \mathrm{~mL}$ of $1.0 \mathrm{~N} \mathrm{NH}_{4} \mathrm{OH}$ and $3 \mathrm{~mL}$ methanol and stored at $-20^{\circ} \mathrm{C}$. All working standard solutions were freshly prepared from the stock before each analytical run. Calibrating solutions were freshly prepared at a scalar concentration by diluting stock solutions in methanol 50\%. This solution is later diluted in whole blood to produce desired concentrations. The calibration concentrations of 6-MP and 6-MMP ranged from 26 to $1040 \mathrm{ng} / \mathrm{mL}$ and $13-520 \mathrm{ng} / \mathrm{mL}$, respectively.

\section{UPLC instruments and chromatographic conditions}

Chromatographic analysis was performed using a water acquity UPLC system consisting of a quaternary solvent manager (Acquity UPLC H-class), sample manager FTN (Acquity UPLC), and TQD detector with ionization source (ZprayTM).

Chromatographic separation was performed using waters acquity UPLC Class BEH C-18 $1.7 \mu \mathrm{m}(2.1 \times 100 \mathrm{~mm})$ column at $35^{\circ} \mathrm{C}$ using column thermostat. The column was protected by a VanGuard ${ }^{\mathrm{TM}}$ Acquity $\mathrm{BEH}$ pre-column. A nitrogen generator compressor (PEAK scientific) was also used. Data were processed using Mass Lynx software. The run was performed with a gradient of two mobile phases consisting of $0.1 \%$ formic acid in water (A) and $0.1 \%$ formic acid in acetonitrile (B) (Table 1 ). The flow rate was $0.2 \mathrm{~mL} /$ minute, and the injection volume was $10 \mu \mathrm{L}$.

The determination of ionization parameters was achieved by multiple reaction monitoring of precursor ions, product ions, and their collision energy parameters (Table 2). The capillary voltage was set to $3.5 \mathrm{kV}$, nitrogen was set as nebulizing gas, $450^{\circ} \mathrm{C}$ was set as desolvation temperature, and $700 \mathrm{~L} / \mathrm{hr}$ as desolvation gas flow. Mass transitions of 6-MP and 6-MMP were monitored at positive ionization and 5-FU at negative ionization. The ionization parameters optimized are shown in Table 2.

\section{DBS sample preparation}

To prepare the blood spots, $40 \mu \mathrm{L}$ of the spiked blood standards of 6-MP and 6-MMP were pipetted and spotted onto CAMAG DBS paper. The papers were then dried for $3.5 \mathrm{hrs}$ at room temperature, cut to a diameter of $8 \mathrm{~mm}$, and placed into a tube. The extraction solution consisted of $4 \mathrm{~mL}$ methanol-acetonitrile (3:1) with $100 \mu \mathrm{L}$ of internal standard 5-FU added to the tube. The tubes were vortex-mixed for 30 seconds and then continued with sonication for 25 minutes at $60^{\circ} \mathrm{C}$. Next, the whole mixture centrifuged for 15 minutes at $3100 \mathrm{rpm}$. The supernatant was later transferred into test tubes and evaporated with nitrogen for 30 minutes at

Table 1: Gradient separation profile

\begin{tabular}{lll}
\hline Time & Mobile phase A (\%) & Mobile phase B (\%) \\
\hline 0.0 & 95 & 5 \\
0.3 & 85 & 15 \\
0.6 & 80 & 20 \\
1.0 & 75 & 25 \\
1.3 & 75 & 25 \\
1.7 & 85 & 15 \\
4.0 & 95 & 5 \\
5.0 & 95 & 5 \\
\hline
\end{tabular}

Table 2: MRM parameters for the analysis of 6-MP, 6-MMP, and 5-FU

\begin{tabular}{llll}
\hline Compound & $\begin{array}{l}\text { Mass } \\
\text { transition }(\mathbf{m} / \mathbf{z})\end{array}$ & Voltage (V) & $\begin{array}{l}\text { Collision } \\
\text { energy }(\mathbf{V})\end{array}$ \\
\hline 6-MP & $153.09>119.09$ & 44 & 20 \\
6-MMP & $167.17>126.03$ & 44 & 20 \\
5-FU & $129.05>42.02$ & 37 & 14 \\
\hline
\end{tabular}

6-MP: 6-mercaptopurine, 6-MMP: 6-methylmercaptopurine,

5-FU: 5-fluorouracil, MRM: Mutiple reaction monitoring $40^{\circ} \mathrm{C}$. The resulting dry extracts were dissolved in $100 \mu \mathrm{L}$ of mobile phase and continued with sonication for 5 minutes. The solution was displaced into auto sampler vials and centrifuged at $3000 \mathrm{rpm}$ for 5 minutes, after which $10 \mu \mathrm{L}$ of the solution was injected into the chromatographic system. The extraction parameters optimized were the amount of extraction solution, sonication temperature, and sonication time.

\section{Determination of lower limit of quantification (LLOQ)}

The LLOQ is the lowest concentration of an analyte in a sample that can be quantified reliably with acceptable accuracy and precision. The mean concentration should be within $20 \%$ of the actual value according to the EMA guidelines.

\section{Selectivity}

Interference from endogen compounds was investigated by analyzing six different sources of the appropriate blank matrix. Blank whole blood was spotted at DBS paper and prepared as above. An absence of interfering components is acceptable where the response is $<20 \%$ of the LLOQ for the analyte.

\section{Linearity}

Calibration curves were measured using a blank sample, a zero sample, and samples at seven concentration levels and then prepared at scalar concentrations. The mean regression coefficients were calculated.

\section{Accuracy, precision, and recovery}

Accuracy and precision of the method were performed intraday and interday. Accuracy and precision were determined at three concentrations, stated as quality control low (QCL), QC medium (QCM), and $\mathrm{QC}$ high $(\mathrm{QCH})$. Accuracy was calculated as the mean percentage deviation from the actual concentration expressed as \% relative error (RE) while precision was expressed by \% relative standard deviation (RSD) calculated. Both parameters should be $\leq \pm 15 \%$ for the QC samples and e $\leq 20 \%$ for LLOQ.

Recovery was performed by comparing the peak areas of extracted DBS samples with those obtained by direct injection of the same amount of drug in standard solutions.

\section{Carry over}

Carry over was assessed by injecting blank DBS samples after a high concentration sample of calibration standard at the upper limit of quantification. Carry over in the blank should not be $>20 \%$ of the LLOQ.

\section{Matrix effect}

Matrix effect was assessed using at least six lots of blank matrix from individual donors. This was achieved by comparing the peak areas in the presence of matrix with those in a pure solution of the analyte. Matrix effect assessment should be performed at QCL and QCH.

\section{Stability assessment}

Stability of analyte in DBS samples was assessed in short-term stability up to $24 \mathrm{hrs}$ at room temperature and long-term stability up to 6 days. Stock solution stability was evaluated comparing the peak areas obtained from direct injection of a diluted solution prepared from the stock stored at $-20^{\circ} \mathrm{C}$ for 16 days with other peak areas obtained from direct injection obtained from a freshly prepared stock.

\section{RESULTS}

\section{Determination of LLOQ}

The LLOQ value of 6-MP obtained was $26 \mathrm{ng} / \mathrm{mL}$ with $\% \mathrm{RE}$ of $-12.76 \%$ to $5.60 \%$ and \% RSD of $6.95 \%$. The LLOQ value of 6-MMP obtained was $13 \mathrm{ng} / \mathrm{mL}$ with $\%$ RE of $-10.92 \%$ to $13.94 \%$ and $\%$ RSD of $10.80 \%$ (Fig. 1).

\section{Selectivity}

Selectivity test was assessed for 6-MP and 6-MMP, respectively, at $26 \mathrm{ng} / \mathrm{mL}$ and $13 \mathrm{ng} / \mathrm{mL}$. No interfering peaks generated from endogenous substances were observed on the chromatograms for blank DBS samples (Fig. 2). 
Linearity

The calibration curve ranges of 6-MP and 6-MMP were $26-1040 \mathrm{ng} / \mathrm{mL}$ and $13-520 \mathrm{ng} / \mathrm{mL}$, respectively. The concentrations made for 6-MP were $26,52,104,208,520,832$, and $1040 \mathrm{ng} / \mathrm{mL}$ and for 6-MMP were $13,26,52,104,208,416$, and $520 \mathrm{ng} / \mathrm{mL}$. The mean regression coefficients obtained for 6-MP and 6-MMP were both 0.999 .

\section{Accuracy, precision, and recovery}

RS error for intraday and interday assay precision was determined by executing three runs on two different days. Four concentrations at LLOQ, QCL, QCM, and QCH were used for both the accuracy and precision tests (Tables 3 and 4). The mean recoveries of 6-MP and
6-MMP with this method were $94.74 \%$ with \% SD 5.70\% and 98.09\% with \% SD 5.32\%

\section{Carry over}

The carry over measurements of 6-MP and 6-MMP after injection of ULOQ compared to the LLOQ concentration were $3.32-19.57 \%$ and $1.83-5.26 \%$, respectively. The carry over of internal standard observed was $0.03-1.90 \%$.

Matrix effect

The matrix effect measurements for 6-MP ranged from $73.73 \%(\mathrm{QCH})$ to $80.79 \%$ (QCL), with \% SD at 6\% and 8\%, respectively, and for 6-MMP from $82.88 \%(\mathrm{QCH})$ to $87.64 \%(\mathrm{QCL})$, at $6 \%$ and $7 \%$

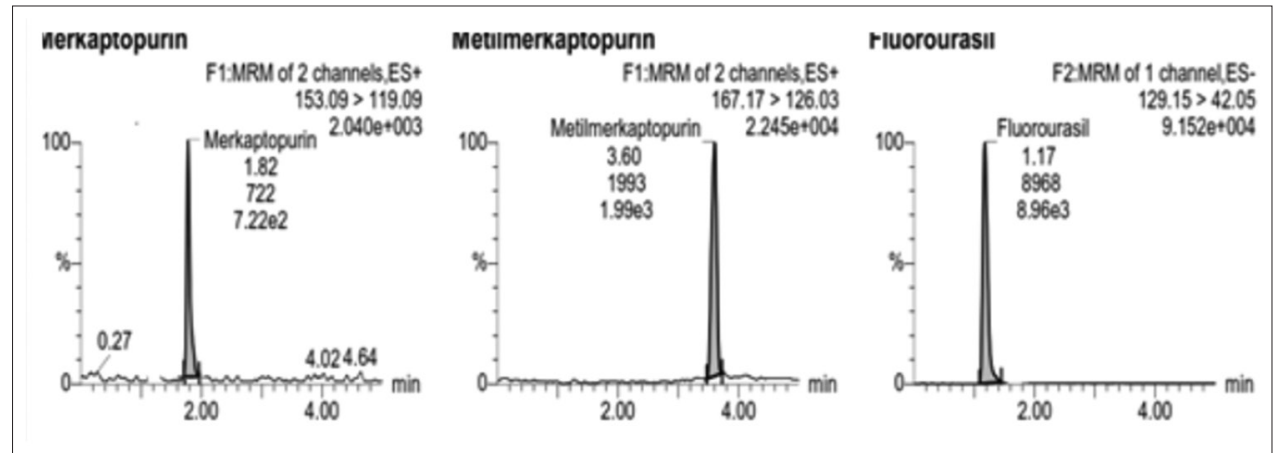

Fig. 1: Lower limit of quantification chromatogram of 6-mercaptopurine (MP), 6-methyl MP
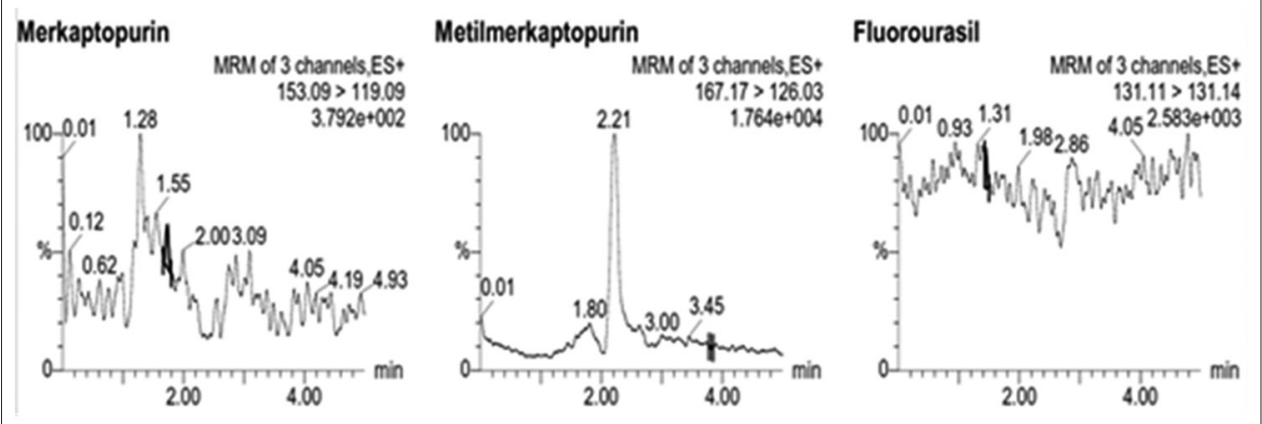

Fig. 2: Blank chromatogram of 6-mercaptopurine (MP), 6-methyl MP

Table 3: Overview of intra and interday accuracy and precision of 6-MP

\begin{tabular}{|c|c|c|c|c|c|c|c|c|c|}
\hline \multirow{2}{*}{$\begin{array}{l}\text { Actual } \\
\text { concentration } \\
(\mathrm{ng} / \mathrm{mL})\end{array}$} & \multicolumn{3}{|l|}{$1^{\text {st }}$ run } & \multicolumn{3}{|l|}{$2^{\text {nd }}$ run } & \multicolumn{3}{|l|}{$3^{\text {rd }}$ run } \\
\hline & $\begin{array}{l}\text { Measured } \\
\text { concentration } \\
(\mathrm{ng} / \mathrm{mL})\end{array}$ & RSD (\%) & RE (\%) & $\begin{array}{l}\text { Measured } \\
\text { concentration } \\
(\mathrm{ng} / \mathrm{mL})\end{array}$ & RSD (\%) & RE (\%) & $\begin{array}{l}\text { Measured } \\
\text { concentration } \\
(\mathrm{ng} / \mathrm{mL})\end{array}$ & RSD (\%) & RE (\%) \\
\hline 26.00 & 24.86 & 6.95 & -4.37 & 28.44 & 5.88 & 9.39 & 22.78 & 5.54 & -12.4 \\
\hline 104.00 & 102.55 & 3.69 & -1.39 & 96.28 & 4.02 & -7.42 & 95.96 & 7.42 & -7.73 \\
\hline 520.00 & 536.74 & 3.91 & 3.22 & 487.29 & 5.29 & -6.29 & 506.39 & 6.37 & -2.62 \\
\hline
\end{tabular}

RSD: Relative standard deviation, RE: Relative error

Table 4: Overview of intra- and interday accuracy and precision of 6-MMP

\begin{tabular}{|c|c|c|c|c|c|c|c|c|c|}
\hline \multirow{2}{*}{$\begin{array}{l}\text { Actual } \\
\text { concentration } \\
(\mathrm{ng} / \mathrm{mL})\end{array}$} & \multicolumn{3}{|l|}{$1^{\text {st }}$ Run } & \multicolumn{3}{|l|}{$2^{\text {nd }}$ Run } & \multicolumn{3}{|l|}{$3^{\text {rd }}$ Run } \\
\hline & $\begin{array}{l}\text { Measured } \\
\text { concentration } \\
(\mathrm{ng} / \mathrm{mL})\end{array}$ & RSD (\%) & RE (\%) & $\begin{array}{l}\text { Measured } \\
\text { concentration } \\
(\mathrm{ng} / \mathrm{mL})\end{array}$ & RSD (\%) & RE (\%) & $\begin{array}{l}\text { Measured } \\
\text { concentration } \\
(\mathrm{ng} / \mathrm{mL})\end{array}$ & RSD (\%) & RE (\%) \\
\hline 13.00 & 13.01 & 13.15 & 0.09 & 13.38 & 10.54 & 2.92 & 12.08 & 8.75 & -7.11 \\
\hline 52.00 & 54.18 & 10.43 & 4.20 & 56.48 & 3.92 & 8.62 & 52.29 & 8.83 & 0.56 \\
\hline 260.00 & 262.52 & 7.25 & 0.97 & 253.71 & 3.88 & -2.42 & 267.02 & 4.31 & 2.70 \\
\hline
\end{tabular}

RSD: Relative standard deviation, RE: Relative error 


\section{Stability assessment}

The stock solution did not show degradation after 16 days of storage at $-20^{\circ} \mathrm{C}(+2 \%)$ deviation from freshly prepared stock solution. 6-MP and 6 -MMP in the DBS cards were stable at room temperature for 6 days

\section{DISCUSSION}

The usage of 6-MP as a treatment for ALL in infants is increasing. The effectiveness of this therapy depends on the metabolism of 6-MP through three metabolism pathways, one of which is the enzyme TPMT to form 6-MMP. High concentrations of 6-MMP can cause a hepatotoxic effect, so the evaluation of this concentration in biological fluids can help clinicians in therapy adjustment. Several methods have been developed to determine the amount of 6-MP and its metabolites in plasma and whole blood, but none use DBS samples. This research is believed to be the first attempt to successfully develop an analytical method for 6-MP and 6-MMP in DBS.

A LLOQ for both 6-MP and 6-MMP was achieved by protein precipitation as the extraction method using acetonitrile-methanol as the extraction solvent. An evaporation step following the precipitation was added to concentrate its analyte to increase the analyte response. The high recovery percentages of 6-MP (at 94.74\%) and 6-MMP (98.09\%) show that the extraction method produced high extraction yields. Validation tests were performed and, as reported above, the values of accuracy and precision fulfilled the EMA guideline range of $\leq \pm 15 \%$ for the QC samples and $\leq 20 \%$ for LLOQ. The calibration curves expressed by the mean regression coefficients of 6-MP and 6-MMP were both 0.999, which means the method is linear, precise, and accurate.

The matrix effect values observed (6-MP: 73.73\% for QCH and $80.79 \%$ for QCL, at \%SD 6\% and 8\%, respectively; 6-MMP: 82.88\% for QCH and $87.64 \%$ for QCL, at $\%$ SD $6 \%$ and 7\%) indicate that endogenous compound causes ion suppression, which can interfere with the analyte ionization process; however, the \%SD values were within EMA limits. Both analytes were stable at the storing condition of $-20^{\circ} \mathrm{C}$ for at least 16 days, and the DBS samples were stable for 6 days at room temperature. In addition, our method has the advantages of fast run time ( 5 minutes) and simple sample preparation with protein precipitation. Further studies need to be made, specifically with the application of this method for therapeutic drug monitoring to ALL patients receiving 6-MP as their therapy.

\section{CONCLUSION}

The method developed and validated as described is suitable for the accurate and precise analysis of 6-MP and 6-MMP simultaneously in DBS using UPLC-MS/MS. The DBS sample preparation procedure is simple, involving protein precipitation followed by analyte reconstitution. To our knowledge, this is the first reported attempt to develop and validate an analysis of 6-MP and 6-MMP in DBS sample. The blood volume required was just $\pm 40 \mu \mathrm{L}$. Thus, this method serves as a milestone for application in in vivo studies in ALL patients.

\section{REFERENCES}

1. Lennard L. The clinical pharmacology of 6-mercaptopurine. Eur J Clin Pharmacol 1992;43(4):329-9.

2. Beaumais TA, Fakhoury M, Medard Y, Azougagh S, Zhang D, Yakouben $\mathrm{K}$, et al. Determinants of mercaptopurine toxicity in paediatric acute lymphoblastic leukemia maintenance therapy. Br J Clin Pharmacol 2010;71(4):575-84.

3. Schmiegelow K, Nielsen SN, Frandsen TL, Nersting J. Mercaptopurine/ Methotrexate maintenance therapy of childhood acute lymphoblastic leukemia: Clinical facts and fiction. J Pediatr Hematol Oncol 2014;36(7):503-17.

4. Schmiegelow K, Bretton-Meyer U 6-mercaptopurine dosage and pharmacokinetics influence the degree of bone marrow toxicity following high-dose methotrexate in children with acute lymphoblastic leukemia. Leukemia 2001;15(1):74-9.

5. Kirchherr H, Shipkova M, von Ahsen N. Improved method for therapeutic drug monitoring of 6-thioguanine nucleotides and 6-methylmercaptopurine in whole-blood by LC/MSMS using isotopelabeled internal standards. Ther Drug Monit 2013;35(3):313-21.

6. Déglon J, Thomas A, Mangin P, Staub C. Direct analysis of dried blood spots coupled with mass spectrometry: Concepts and biomedical applications. Anal Bioanal Chem 2012;402(8):2485-98

7. Wilhelm AJ, den Burger JC, Swart EL. Therapeutic drug monitoring by dried blood spot: Progress to date and future directions. Clin Pharmacokinet 2014;53(11):961-73.

8. Evans C, Arnold M, Bryan P, Duggan J, James CA, Li W, et al. Implementing dried blood spot sampling for clinical pharmacokinetic determinations: Considerations from the IQ consortium microsampling working group. AAPS J 2015;17(2):292-300.

9. Hawwa AF, Al Bawab A, Rooney M, Wedderburn LR, Beresford MW, McElnay JC. A novel dried blood spot-LCMS method for the quantification of methotrexate polyglutamates as a potential marker for methotrexate use in children. PLOS One 2014;9(2):e89908. 